\title{
Regulation of ferulic catabolic genes in Pseudomonas fluorescens BF13: involvement of a MarR family regulator
}

\author{
C. Calisti • A. G. Ficca • P. Barghini • M. Ruzzi
}

Received: 21 April 2008 /Revised: 28 May 2008/Accepted: 28 May 2008 / Published online: 25 June 2008

(C) Springer-Verlag 2008

\begin{abstract}
In Pseudomonas fluorescens BF13, the cluster of genes essential for degradation of ferulic to vanillic acid (ech, $v d h$ and $f c s$ ) is expressed in ferulic but not in succinicgrown cells. In the upstream region, we identified a gene, fer $R$, encoding a protein homologous to transcriptional regulators of the MarR family. A ferR knockout mutant (BF13-89) showed a 3.5-fold increase in expression of an ech-reporter gene fusion compared with the parent strain in succinic-grown cells, indicating that the fer $R$ gene product negatively regulates expression of the ferulic catabolic operon in P. fluorescens BF13. Consistent with the increased expression of the catabolic genes in the ferR mutant, BF13-89 showed a shorter (relative to its FerR $^{+}$ parent) lag phase during carbon source shift from succinic to ferulic acid. However, expression of ech-lacZ fusion did not increase in BF13-89 grown in the presence of ferulic acid, indicating that FerR has a second function as transcriptional activator. Expression of ech-lacZ in a feruloyl-CoA synthetase-deficient strain revealed unambiguously that FerR-mediated activation of the ferulic catabolic operon is dependent on the thioester product of the feruloyl-CoA synthetase reaction.
\end{abstract}

C. Calisti and A.G. Ficca contributed equally.

C. Calisti P. Barghini · M. Ruzzi $(\square)$

Department of Agrobiology and Agrochemistry, University of Tuscia,

via C. de Lellis snc,

01100 Viterbo, Italy

e-mail: ruzzi@unitus.it

A. G. Ficca

Department of Scienze Ambientali, University of Tuscia, 01100 Viterbo, Italy
Keywords Ferulic acid catabolism · Pseudomonas . MarR family regulator $\cdot$ FerR $\cdot$ CoA thioester .

Gene transcription

\section{Introduction}

Ferulic acid (4-hydroxy-3-methoxycinnamic acid) is a ubiquitous plant constituent that is produced from the metabolism of phenylalanine and tyrosine. It is the main phenolic component found in cell walls of monocotyledons constituting about $1.4 \mathrm{~g} / \mathrm{kg}$ in barley grains (Nordkvisk et al. 1984), $9 \mathrm{~g} / \mathrm{kg}$ in rice endosperm cell wall (Shibuya 1984), $6.6 \mathrm{~g} / \mathrm{kg}$ in wheat (Smith and Hartley 1983), and $31.0 \mathrm{~g} / \mathrm{Kg}$ in maize bran (Saulnier et al. 1995).

There is a growing interest in the potential use of ferulic acid as feedstock for biocatalytic conversion into valuable compounds (Rosazza et al. 1995). Specifically, there is significant interest in microbial pathways of ferulic acid dissimilation that generate aldehyde intermediates, such as vanillin, the main flavour component of vanilla (Walton et al. 2000; Priefert et al. 2001; Schrader et al. 2004).

Six major pathways have been proposed for the initial degradation of ferulic acid, namely non-oxidative decarboxylation, side-chain reduction, coenzyme-A-independent and coenzyme-A-dependent deacetylation, demethylation and oxidative coupling (as reviewed in Mathew and Abraham 2006). The genes and enzymes involved in the coenzyme-A-dependent, non- $\beta$-oxidative pathway, which is the most common pathway of ferulic acid degradation in bacteria (Priefert et al. 2001), have been characterized in Pseudomonas fluorescens AN103 (Gasson et al. 1998), Pseudomonas sp. HR199 (Overhage et al. 1999), Pseudomonas putida KT2440 (Plaggenborg et al. 2003), Streptomyces setonii (Muheim and Lerch, 1999), Amycolatopsis 